\section{Going after GEFs in cancer}

\section{By Tim Fulmer, Senior Writer}

Despite the association between mutations in Rho guanine nucleotide exchange factors and cancer, no companies are pursuing GEF inhibitors. Antagonizing the targets is challenging because it requires inhibiting a protein-protein interaction, a task for which small molecules are not well suited. Also, GEFs are intracellular and therefore not easily accessible to antibody therapeutics.

Now, a team of French researchers has designed a peptide inhibitor of TGAT, a GEF implicated in T cell leukemia. ${ }^{.}$The peptide has shown efficacy in vivo, although it may need to be shortened to better permeate cell membranes.

Rho guanine nucleotide exchange factors (ARHGEFs; GEFs) bind to and activate Rho family GTPases to trigger cellular processes like cell migration and proliferation. Mutations in GEFs can cause aberrant activation of GTPases with consequent malignant transformation. ${ }^{2}$

The team at the Macromolecular Biochemistry Research Center (CRBM) had previously used an aptamer-based screen to identify a 42-amino-acid peptide dubbed TRIP $\alpha$ that bound a nonmutant triple functional domain (PTPRF interacting) (TRIO; TGAT) and prevented it from interacting with RhoA GTPase in healthy cells. ${ }^{3}$

In their current Chemistry \& Biology paper, the researchers generated a second library of peptide aptamers based on TRIP $\alpha$ that were screened for their ability to bind and inhibit a mutant of TGAT associated with leukemia.

Two 42-amino-acid peptides, TRIP ${ }^{\mathrm{E} 2 \mathrm{G}}$ and TRIP ${ }^{\mathrm{T} 16 \mathrm{M} / 1 \mathrm{117}}$, inhibited the oncogenic TGAT in vitro and in cultured fibroblasts. In mice engrafted with cells expressing mutated Tgat, expression of TRIP ${ }^{\mathrm{E} 32 \mathrm{G}}$ in the same cells significantly decreased tumor weight compared with that seen in control mice that did not express $\operatorname{TRIP}^{\mathrm{E} 32 \mathrm{G}}(p=0.019)$.

CRBM is jointly run by the Centre National de la Recherche Scientifique (CNRS), University of Montpellier I and University of Montpellier II.

\section{Prepping the peptide}

The TGAT-targeting peptides will likely require further optimization to increase their stability and deliverability.

"The paper addresses the basic challenge of identifying a protein-protein interaction that may be usefully targeted by inhibitors to treat some forms of adult leukemia," said Paul Ko Ferrigno, a senior lecturer at the

\section{Leeds Institute of Molecular Medicine.}

The challenge, added Ko Ferrigno, is that the inhibitors are "still too long for inexpensive chemical synthesis." A first priority should be figuring out how to shorten the peptide without impairing its activity, he said.

Another hurdle will be delivering the peptide across the cell membrane, said Bernd Groner, director of the Georg Speyer Haus of the Institute for Biomedical Research. He said peptide stability and bioavailability will also have to be addressed.

Anne Debant, a corresponding author on the paper and researcher at CRBM, acknowledged that the inhibitory peptides will require further optimization. "The 42 -amino-acid length is potentially a problem for peptide synthesis and perhaps also for stability within the cell. The shorter the peptide, the better," she said.

Once a peptide of optimal length is identified, Debant said the group will consider two ways of getting it into cells: fusing the peptide to a second, cell-permeable peptide or using a lentiviral vector that carries a gene encoding the peptide.

Ko Ferrigno thinks the results in Chemistry \& Biology could open the door to developing small molecules targeting the GEF-GTPase interaction. The idea, he said, is to "seek small molecules that mimic the peptide."

That could be done by using structural studies to guide the synthesis of small molecules "or more empirically by screening for small molecules that displace the peptide from the targeted protein-protein interface," he said.

Indeed, the CRBM researchers are keeping the door open to small molecules. "On a longer time scale, it may be possible to use a peptide aptamer displacement screen to identify a small molecule that has exactly the same inhibitory properties as the initial 42 -amino-acid peptide," said Susanne Schmidt, also a corresponding author on the paper and a researcher at CRBM.

Schmidt said an application for an international patent covering the paper's findings has been filed by CNRS. The application is unlicensed.

Fulmer, T. SciBX 2(19); doi:10.1038/scibx.2009.775

Published online May 14, 2009

\section{REFERENCES}

1. Bouquier, N. et al. Chem. Biol.; published online April 24, 2009; doi:10.1016/j.chembiol.2009.02.006

Contact: Anne Debant, University of Montpellier, Montpellier, France e-mail: anne.debant@crbm.cnrs.fr

Contact: Susanne Schmidt, same affiliation as above e-mail: Susanne.schmidt@crbm.cnrs.fr

2. Rossman, K. et al. Nat. Rev. Mol. Cell Biol. 6, 167-180 (2005)

3. Schmidt, S. et al. FEBS Lett. 523, 35-42 (2002)

COMPANIES AND INSTITUTIONS MENTIONED

Centre National de la Recherche Scientifique, Paris, France Georg Speyer Haus of the Institute for Biomedical Research, Frankfurt am Main, Germany

Leeds Institute of Molecular Medicine, Leeds, U.K.

Macromolecular Biochemistry Research Center, Montpellier, France

University of Montpellier I, Montpellier, France

University of Montpellier II, Montpellier, France 